\title{
Neural Illustration based Creature Recognition System
}

\author{
Prabakaran.M ${ }^{2}$ and Dr.T.Senthilkumar ${ }^{1}$ \\ Reader ${ }^{1}$, Research Scholar ${ }^{2}$ \\ ${ }^{1}$ Head, Dept of Automobile Engineering \\ ${ }^{1}$ Anna University- Tiruchirappali, \\ ${ }^{1}$ Tiruchirappali, Tamil Nadu. \\ ${ }^{2}$ Vinayaka Missions University, \\ Salem, Tamil Nadu.
}

\begin{abstract}
Face detection is one of the challenging problems in image processing. In this paper we have proposed a new technology named neural GUI based human recognition robotics. This system contains the skin color which is the main feature of faces for detection, and then the skin face candidate is examined by using the neural GUI, which learn from the feature of faces to classify whether the original image includes a face or not. Finally the classification is based on GUI approach. The experiment results on upright frontal color face images from the internet show an excellent detection rate.
\end{abstract}

\section{General Terms}

Face detection, Segmentation

\section{Keywords}

Skin color segmentation, compressed domain, Neural Networks.

\section{INTRODUCTION:}

Face detection is an active area of research spanning disciplines such as image processing, pattern recognition and computer vision. The face detection and recognition are the two preliminary steps in wide applications such as personal identity, video surveillance etc. The detection efficiency influences the performance of these systems; there have been various approaches for face detection, which classified into four categories
(i) Knowledge based method
(ii) Feature based method
(iii) Template matching method
(iv) Appearance based method

In the compressed domain chrominance, shape and DCT information coefficient was combined by Wang and Chang to achieve high-speed face detection without decoding of the compressed video image. Now our technology has improved with the recognition of face with the visual GUI based recognition. This approach will reduce the code complexity in detection area .while other detection method has lot of algorithm complexity as well as coding complexity.
The objectives of this research are to develop better recognition method and also aim to improve the segmentation that will assist in quick detection of faces from images and also to implement a classifier face based on image for face detection. Most of the interested readers are referred to the comprehensive survey on face detection by Yang et al, and by Hjelmas and Low. The new algorithms introduced combines two methods to perform fast and accurate face detection system, which are a feature based method and image based method. The feature based method uses a pre-processor of the image based method and guides the search of image based methods using GUI that examine the face candidate regions instead of performing huge search in every part of the test image. Hwei proposed Extraction regions of skin can be either pixel-based or region based .The diagram of our proposed techniques is presented.

\section{FACE DETECTION IN GUI}

Many techniques for face detection in image were classified into four categories. Knowledge based method depends on using the rules about human facial feature .It is easy to come up with simple rules to describe the features of a face and their relationships. For example, a face often appears in an image with two eyes that are symmetric to each other, a nose, and a mouth and feature's relative distance and position represent relationships between features. After detecting features, verification is done to reduce false detection. This approach is good for frontal image; the difficulty of it is how to translate human knowledge into Known rules and to detect faces in different poses.

\subsection{Illustration GUI based Method}

In this approach, there is a predefined standard face pattern is used to match with the pixels in the image to determine whether they are faces or not. It uses training algorithms to classify regions into face or non-face classes. Image-based techniques depends on multi-resolution window scanning to detect faces, so these techniques have high detection rates but slower than the feature-based techniques. Eigen-faces and neural networks are examples of image-based techniques. This approach has advantage of being simple to implement, but it cannot effectively deal with variation in scale, pose and shape. 


\subsection{Next Generation based Method}

This approach depends on extraction of facial features that are not affected by variations in lighting conditions, pose, and other factors. These methods are classified according to the extracted features [3]. Feature-based techniques depend on feature derivation and analysis to gain the required knowledge about faces. Features may be skin color, face shape, or facial features like eyes, nose, etc. Feature based methods are preferred for real time systems where the multi-resolution window scanning used by image based methods are not applicable. Human skin color is an effective feature used to detect faces, although different people have different skin color. Several studies have shown that the basic difference is based on their intensity rather than their chrominance. Textures of human faces have a special texture that can be used to separate them from different objects. Facial Features method depends on detecting features of the face. Some users use the edges to detect the features of the face, and then grouping the edges. Some others use the blobs and the streaks instead of edges. For example, the face model consists of two dark blobs and three light blobs to represent eyes, cheekbones, and nose. The model uses streaks to represent the outlines of the faces like, eyebrows, and lips. Multiple Features methods use several combined facial features to locate or detect faces. First step is finding the face by using features like skin color, size and shape and then verifying these candidates using detailed features such as eye brows, nose, and hair.

\subsection{Guide Alike Scheme}

Guide alike scheme uses the correlation between pattern in the input image and stored standard patterns of a whole face / face features to determine the presence of a face or face features. Predefined templates as well as deformable templates can be used.

\section{FACADE REVEALING ALGORITHMS}

Information of skin color in a color image is a very popular and useful feature for face detection. The obvious advantage of this method is simplicity of skin detection rules that leads to construction of a very rapid classifier. We can use color information as a feature to identify a person's face in an image because human faces have a special color distribution that differs significantly, although not entirely, from those of the background objects. Previous studies have found that pixels belonging to skin region exhibit similar chrominance components within and across different human races. In the YCbCr color space, chrominance components are represented by $\mathrm{Cb}$ and $\mathrm{Cr}$ values. Thus, skin color model can be derived from these values. By using threshold techniques, skin color pixels are identified by the presence of a certain set of $\mathrm{Cb}$ and $\mathrm{Cr}$ values which corresponding to the respective ranges of $\mathrm{RCb}$ and $\mathrm{RCr}$ values of skin color. Otherwise, the pixel is classified as non skin color. The system being designed into three main categories, preprocessing, segmentation, classification using neural Networks

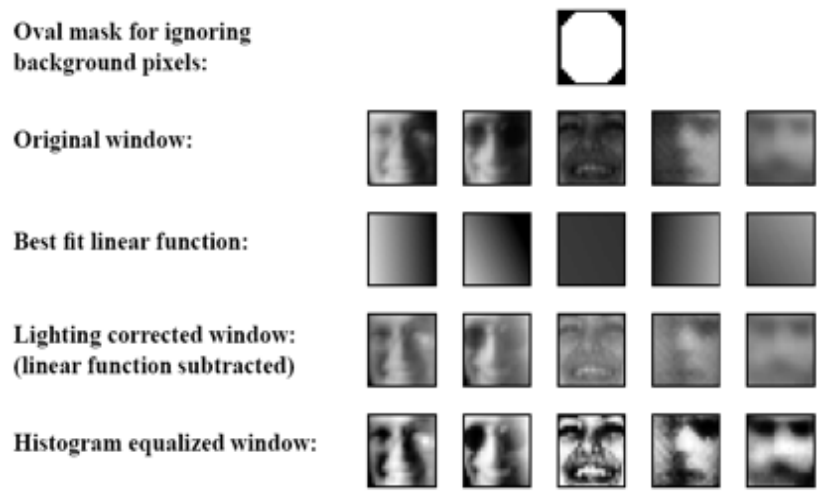

Fig 1: Mask and histogram equalized windows

\subsection{Preprocessing}

In fact, processing skin color is faster than other facial features collecting a data set of skin face by cropping or cutting manually the image skin face and non-skin face to get a dataset of face and non-face. Different people have different skin color, while the difference lies mostly in the color intensity not in chrominance color itself. Literature survey shows that $\mathrm{Y} \mathrm{Cb} \mathrm{Cr}$ color space is one of the successful color spaces in segmenting skin color accurately. To select the suitable color space to model skin color and a void variation, of lighting condition, $\mathrm{Cb}$ and $\mathrm{Cr}$ Color space, Extract DCT coefficient features from $\mathrm{Cb}$ and $\mathrm{Cr}$ blocks.

\subsection{Segmentation Casing Shade}

Skin color information is very important features for many researches; however the accuracy of skin color detection is important for face detection [2]. In this paper we convert the image from $\mathrm{RGB}$ to $\mathrm{YCbCr}$, whereas $\mathrm{RGB}$ is sensitive to the variation of intensity. Many skin detection methods ignore the luminance component of the color space, to achieve independent model of the differences in skin appearance that may arise from the difference of human race, and also reduce the space dimension. After collecting different human faces and analyzing the histogram distribution, sample skin color values of chrominance component are used to represent the likelihood of the pixel belonging to the skin region. It was found that the chrominance component of the skin color fails in a certain range. $\mathrm{X}$ is skin color [3], if its projection on the $\mathrm{Cb}$ and $\mathrm{Cr}$ plane is inside predetermined rectangle $C b \in R_{c b}$ and $C r \in R_{c r}$ i.e., $C_{r 1} \leq C_{r} \leq C_{r 2}$ and $C_{b 1} \leq C_{b} \leq C_{b 2}$ where $R_{c b}=\left[C_{b 1}, C_{b 2}\right]$ and $R_{c r}=\left[C_{r 1}, C_{r 2}\right.$ ], which are found experimentally used to eliminate quickly non-skin face color and also to improve the segmentation of s kin color regions. The mask and histogram equalized windows are shown in figure 1 . 


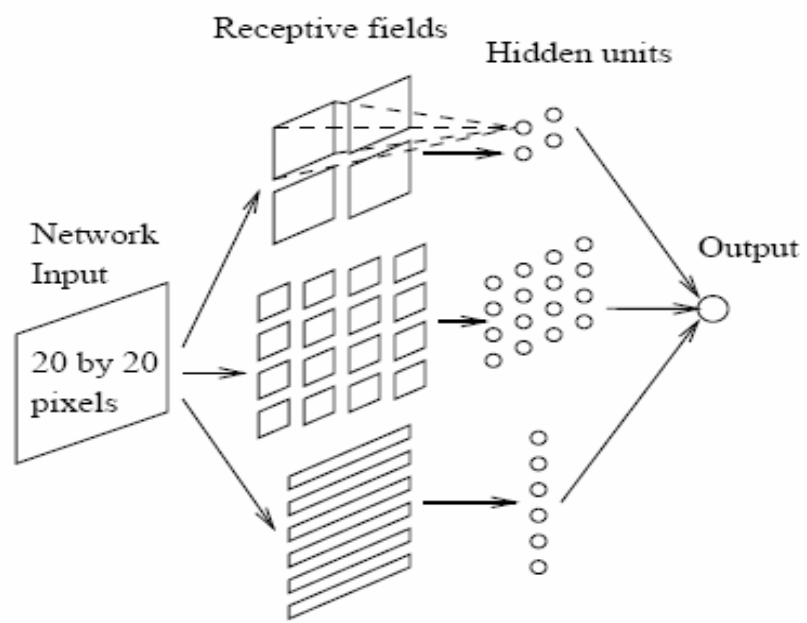

Fig 2: Neural Network Architecture

\subsection{Characteristic Mining}

Discrete Cosine Transform is used widely in many applications and mainly used in the compressed data domain and forms the basis well known JPEG image compression format. Jiang el.al [1] introduced simple low cost and fast algorithms that extract dominant color feature directly for DCT rather than in the pixel domain. The extracted DCT Coefficient can be used as type of a signature of which might be useful for recognition task, such as facial expression recognition [5]. The proposed technique derived from [1], The system calculates the 2D-DCT for each cropped skin block coming out of the previous stage. This result in a matrix of $1 \times 48$ coefficients of both $\mathrm{Cb}$ and $\mathrm{Cr}$ color space components within the processed image block, these values are taken to construct the feature vector. Empirically, the upper left corner of the 2D-DCT matrix contains the most important values, because they correspond to lowfrequency, however the upper most coefficient is called DC and it correspond to average light intensity of the block. The others are called AC, and those coefficients provide useful information about the texture detail in the blocks. For each block we use the DC's and the first three zig zag order AC's as a set of $1 \times 4$ vector coefficients.

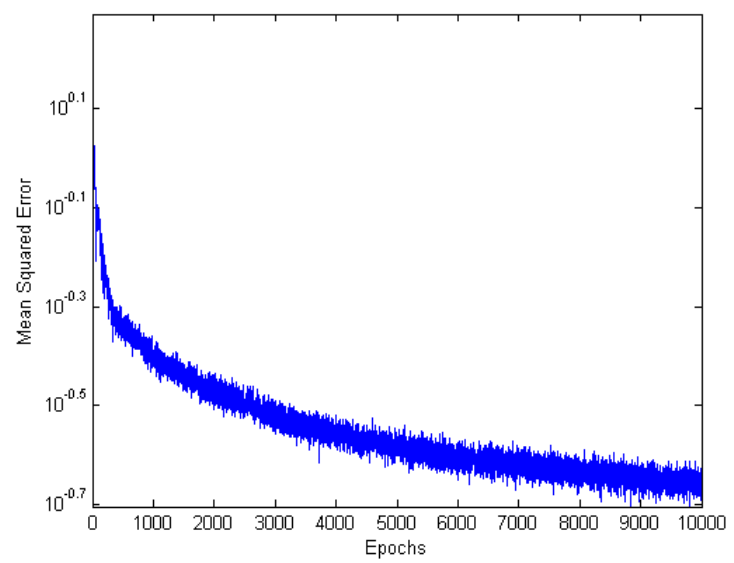

Fig 3: Mean squared error during training

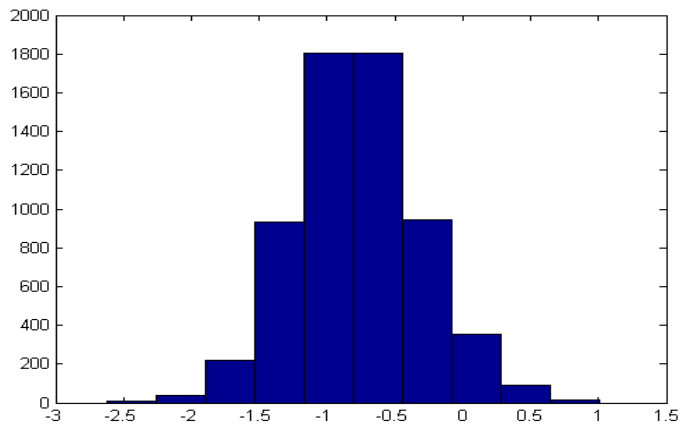

Fig 4: Histogram of the classified training images (only non-faces)

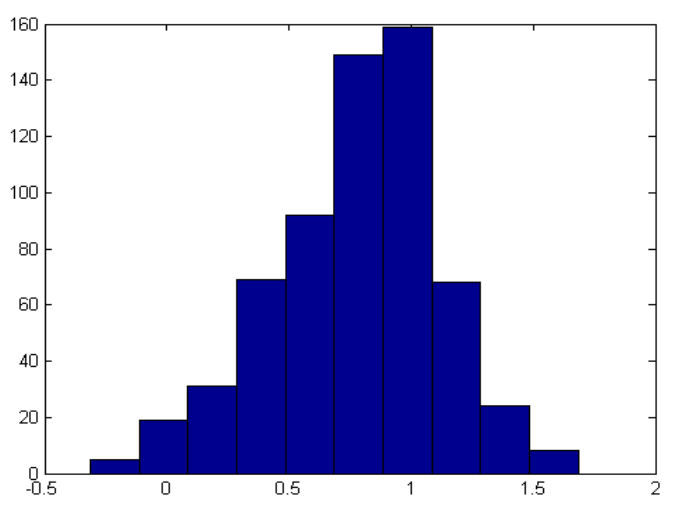

Fig 5: Histogram of the classified training images (only faces)

\subsection{Classification}

Neural networks are often used in face detection, Rowley, Baluja, Kanade [4] proposed a face detection methods based on neural networks that could discriminate between face and non face on large dataset images. In our system, we use Multi Layer Perception (MLP) Back Propagation Neural Networks in order to train data set and classify features that are extracted using DCT (Discrete Cosine Transform Coefficient). The Neural Network architecture is shown in figure 2. After dividing into blocks of size $8 \times 8$ pixels, Training using a vector obtained from $18 \times 27$ training data set of $8 \times 8$ pixel block for true oval face may usually guarantees that only pixels the face are used as input to neural networks, however, to produce an output of 0.9 for the skin face and 0.1 for the non-skin face after repeatedly presented input samples and desired targets, compared the output with the desired and measuring the error and adjusting the weights until correct output for every input[4]. The main advantage of choosing Back Propagation Neural Networks is the simplicity and capability in supervised pattern matching. The mean squared error during training of Neural Networks is shown in figure 3 . The histograms of the classified training images for nonface and face images are shown in figures 4 and 5 respectively. 


\section{GUI SCHEMA}

Neural networks have been applied in many pattern recognition problems like object recognition. There is many image based face detection using neural networks the most successful system was introduced by Rowley et al [4] as using skin color segmentation to test an image and classify each DCT based feature vector for the presence of either a face or non face. The GUI used in this paper is Back Propagation Neural Networks and was chosen because of simplicity and its capability in supervised pattern matching. The structure of the neural network with three layers, the input layer is a vector of $1 \mathrm{x} \mathrm{n} \mathrm{DCT}$ coefficient vectors of neuron from each image either face or non face image, the hidden layers has $\mathrm{n}$ neurons, and the output layer is a single neuron which is 0.9 if the face is presented and 0.1 otherwise. The neural networks is trained using DCT coefficient feature vectors after skin face color candidate obtained from the segmentation stage, which are the DC and the first three zig zag order AC's features samples from each blocks $8 \times 8$ pixels of an manually cropped image $18 \times 27$ pixel of face and non- faces to classify each feature vector as output value 0.9 for a face and 0.1 for nonface

\section{EXPRIMENTS AND RESULTS}

We show in this section a set of experimental results to present the performance of the proposed system, the experiment was implemented using Matlab Version 7.2 on the Intel Pentium(4) 2.80Ghz 1.00GB of RAM and Windows XP operating system. This section presents results of experiment applied on the unknown input test image containing a face or non-face. Starting with sliding overlapping window $18 \times 27$, by overlap scanning the window, where different overlap parameter used 1,2 up to the half pixels, in our experiment 9 pixel is the half of the window it might be maximum overlap, then each part of the unknown test image is scanned using slide window and extracted the DCT features and feed it to the trained neural networks of the dataset of images. However the neural networks tested with the trained neural networks and classify it to see if the part containing a face or non face.The experiment results shows that our face detection system is reliable that neural networks are able to detect and classify pattern features accurately under different overlap sliding scan window over the unknown input test image. The convergent response of training dataset shows accurate and excellent face and non classification. The next step is to perform some analysis of the network response. By putting the entire data set through the network (training, validation and test) to perform a linear regression between the network outputs and the corresponding targets, according to the excellent response of the Back Propagation Neural Networks with the target desired, the classification performance provides a comprehensive excellent picture of the classification performance of the classifier as shown in figure 6 and table 1 .

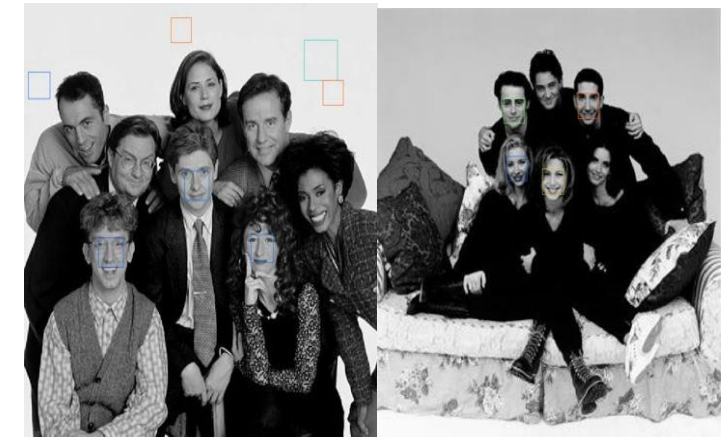

Fig 6: Figure with mask for classifying the face and nonface images

Table 1: The experimental results

\begin{tabular}{|c|c|c|}
\hline System & Detect Rate & False Detect Rate \\
\hline $\begin{array}{c}\text { My network } \\
\text { (27 hidden nodes, 1527 } \\
\text { connections) }\end{array}$ & $41.3 \%$ & $1 / 227580$ \\
\hline $\begin{array}{c}\text { Paper's network 1 } \\
\text { (52 hidden nodes, 2905 } \\
\text { connections) }\end{array}$ & $90.9 \%$ & $1 / 98459$ \\
\hline $\begin{array}{c}\text { Paper's network 2 } \\
\text { (78 hidden nodes, 4357 } \\
\text { connections) }\end{array}$ & $89.5 \%$ & $1 / 115576$ \\
\hline $\begin{array}{c}\text { Paper's network 3 } \\
\text { (52 hidden nodes, 2905 } \\
\text { connections) }\end{array}$ & $89.5 \%$ & $1 / 85230$ \\
\hline $\begin{array}{c}\text { Paper's network 4 } \\
\text { (78 hidden nodes, 4357 } \\
\text { connections) }\end{array}$ & $90.7 \%$ & $1 / 78992$ \\
\hline
\end{tabular}

\section{CONCLUSIONS}

This paper proposes a new algorithm for face detection in the compressed domain, extracted DCT coefficient vector features after segmentation a face skin candidate using skin color information on both $\mathrm{Cb} \mathrm{Cr}$ color space, along with Back Propagation Neural Networks classifier. We have divided the problem into three stages pre-processing, segmentation, and classification using Back Propagation Neural Networks. The system has been tested on a dataset of upright frontal color face images from the internet and achieved excellent detection rate. 


\section{REFERENCES}

[1] Jiamin Jiang, Ying Weng,and P.LI “ Dominant colour extraction in DCT Domain" in Image and Vision computing 24, 2002 published by Elsevier B.V,pp.129177.

[2] Hwei-Jen Lin, Shu-Yi Wang, Shwu-Huey, and Yang-TaKao "Face Detection Based on Skin Color Segmentation and Neural Network" IEEE Transactions on, Volume: 2, pp544- 549, ISBN: 0-7804-9422-4

[3] Y. Ming-Hsuan, D. J. Kriegman, and N. Ahuja, "Detecting faces in Images: a survey," Pattern Analysis and Machine Intelligence, IEEE Transactions on, vol. 24, pp. 44-58, 2002.

[4] H. A. Rowley, S. Baluja, and T. Kanade, "Neural networkbased face detection," in Pattern Analysis and Machine Intelligence, IEEE Transactions on, vol. 20, 1998, pp. 24-48.

[5] L. Ma, Y. Xiao, K. Khorasani, and R. K. Ward, "A new facial expression recognition technique using 2D DCT and k-means algorithm", in Proc.International Conference on Image Processing,Oct. 2004, pp. 129172.

[6] F.Smach, M.Atri, J.Miteran and M.Abid "Design of a Neural Networks Classifier for Face Detection" in Journal of computer Science 2(3):pp257-260, 2006

[7] Lamiaa Mostafa, Sharif Abdelazeem "Face Detection Based on Skin Color Using Neural Networks" in GVIP 05 Conference, pp19-21, Dec 2006, CICC, Cairo, Egypt.

[8] V. Vezhnevets, V. Sazonov, and A. Andreeva, "A survey on pixel-based skin color detection techniques", in Proc. Graphicon-2003
[9] H. Kruppa, M. A. Bauer, and B. Schiele, "Skin patch detection in Real world images", in Proc. of the DAGMSymposium, 2002, pp. 109-116.

[10] L. Ma, Y. Xiao, K. Khorasani, and R. K. Ward, "A new facial expression recognition technique using 2D DCT and k-means algorithm", in Proc.International Conference on Image Processing, Oct. 2004, pp. 12691272.

[11] L. Ma and K. Khorasani, "Facial expression recognition using constructive feed forward neural networks", IEEE Transactions on Systems,Man and Cybernetics, Part B, Vol. 34, No. 3, June 2004, pp.1588 - 1595

[12] L. Ma, Y. Xiao, K. Khorasani, and R. K. Ward, "A new facial expression recognition technique using 2D DCT and k-means algorithm", in Proc.International Conference on Image Processing,Oct. 2004, pp. 12691272.

[13] Jiamin Jiang,Ying Weng,and P.LI “ Dominant colour extraction in DCT Domain" in Image and Vision computing 24, 2006 published by Elsevier B.V,pp.12691277.

[14] E. Hjelmås, and B. K. Low, "Face detection: a survey", Computer Vision and Image Understanding, Vol. 83, No. 3, Sept. 2001, pp. 236-274.

[15] H. Wang and S. -F. Chang, "A highly efficient system for automatic face region detection in mpeg video," In IEEE Trans. CSVT, 7(4), 1997. 